\title{
Intracerebral Hemorrhage
} with Intraventricular Extension Associated with Loss of Consciousness at Symptom Onset

\author{
Jens Witsch ${ }^{1,2}$, Guido J. Falcone ${ }^{1}$, Audrey C. Leasure ${ }^{1}$, Charles Matouk ${ }^{3}$, Matthias Endres ${ }^{4}$, Lauren Sansing ${ }^{1}$,
} Daniel Woo ${ }^{5}$ and Kevin N. Sheth ${ }^{1 *}$ (D)

(c) 2021 The Author(s)

\begin{abstract}
Background: In patients with spontaneous intracerebral hemorrhage (ICH), pre-hospital markers of disease severity might be useful to potentially triage patients to undergo early interventions.

Objective: Here, we tested whether loss of consciousness (LOC) at the onset of ICH is associated with intraventricular hemorrhage (IVH) on brain computed tomography (CT).

Methods: Among 3000 ICH cases from ERICH (Ethnic/Racial Variations of Intracerebral Hemorrhage study, NS069763), we included patients with complete $\mathrm{ICH} / \mathrm{IVH}$ volumetric $\mathrm{CT}$ measurements and excluded those with seizures at ICH onset. Trained investigators extracted data from medical charts. Mental status at symptom onset (categorized as alert/ oriented, alert/confused, drowsy/somnolent, coma/unresponsive/posturing) and 3-month disability (modified Rankin score, mRS) were assessed through standardized interviews of participants or dedicated proxies. We used logistic regression and mediation analysis to assess relationships between LOC, IVH, and unfavorable outcome (mRS 4-6).

Results: Two thousand seven hundred and twenty-four patients met inclusion criteria. Median admission Glasgow Coma Score was 15 (interquartile range 11-15). 46\% had IVH on admission or follow-up CT. Patients with LOC (mental status: coma/unresponsive, $n=352$ ) compared to those without LOC (all other mental status, $n=2372$ ) were younger ( 60 vs. 62 years, $p=0.005$ ) and had greater IVH frequency (77 vs. 41\%, $p<0.001$ ), greater peak ICH volumes (28 vs. $11 \mathrm{ml}, p<0.001$ ), greater admission systolic blood pressure (200 vs. $184 \mathrm{mmHg}, p<0.001$ ), and greater admission serum glucose (158 vs. $127 \mathrm{mg} / \mathrm{dl}, p<0.001)$. LOC was independently associated with IVH presence (odds ratio, OR, 2.6, Cl 1.9-3.5) and with unfavorable outcome (OR 3.05, Cl 1.96-4.75). The association between LOC and outcome was significantly mediated by IVH (beta $=0.24$, bootstrapped Cl $0.17-0.32$ ).
\end{abstract}

Conclusion: $\mathrm{LOC}$ at ICH onset may be a useful pre-hospital marker to identify patients at risk of having or developing $\mathrm{IVH}$.

Keywords: Intracerebral hemorrhage, Intraventricular hemorrhage, Cohort studies, Patient outcome assessment

*Correspondence: kevin.sheth@yale.edu

1 Department of Neurology, Yale School of Medicine, 15 York Street, LLCI 10th floor, Room 1003C, P.O. Box 208018, New Haven, CT 06520, USA

Full list of author information is available at the end of the article

\section{Introduction}

Spontaneous intracerebral hemorrhage $(\mathrm{ICH})$ is a devastating neurological disease. One-year mortality is close to $50 \%$ [1], and the incidence of ICH has nearly tripled over the past 70 years to most recently 73 cases per 100,000 person-years [2]. New treatment targets and effective therapies are urgently needed. 
Intraventricular hemorrhage extension (IVH) of $\mathrm{ICH}$ is independently associated with unfavorable outcome [3-5]. Factors that are thought to mediate the worsening effect on outcome are meningitis, induced by blood breakdown products, development of hydrocephalus, and the excess intracranial volume itself leading to increased intracranial pressure [6,7]. An analysis of the INTERACT-2 study cohort showed that IVH was independently associated with both early (within $24 \mathrm{~h}$ ) and late (up to 7 days) neurological deterioration after ICH [8]. Thus, early recognition of IVH might constitute the first step toward establishing new therapies aiming at containment or removal of intraventricular blood, which might then avert such secondary worsening.

IVH is time-sensitive and associated with hematoma expansion (HE) [4,5,9-11], which in turn can be restricted through blood pressure control, manipulation of coagulation parameters, or surgical intervention $[12,13]$. In $48 \%-54 \%$ of cases, IVH develops in the first few hours after symptom onset and is already present on hospital admission computed tomography (CT) scan $[4,5]$. Thus, determination of IVH risk is more likely to impact patient triage, acute management, and potential surgical prearrangements if it happens before arrival at the receiving hospital. Pre-hospital IVH markers may become clinically relevant in the context of mobile stroke units where a CT diagnosis of a developing intracranial hemorrhage can be made before hospital arrival [14]. Alternatively-in the absence of a diagnostic CT scan before hospital arrival-pre-hospital markers may serve as part of diagnostic panels, in conjunction with acute serum biomarkers aiming to distinguish between ischemic and hemorrhagic stroke [15-17].

In patients with spontaneous subarachnoid hemorrhage, loss of consciousness (LOC) at ictus was found to be associated with greater amounts of subarachnoid blood [18]. We speculated that intraventricular extension of ICH might be associated with LOC, too, possibly through a sudden increase in intracranial pressure from blood pouring into the ventricular space rather than being retained by brain tissue. Here, we tested whether LOC at the onset of ICH might serve as an acute clinical marker of IVH on admission or follow-up CT.

\section{Methods}

\section{Patient Selection and Data Collection}

We present a retrospective analysis of the data collected in the Ethnic/Racial Variations of Intracerebral Hemorrhage (ERICH) study. Methods of the ERICH study have been described in detail previously [19]. In brief, ERICH is a multicenter, prospective case-control study that recruited 1000 non-Hispanic Black people, 1000 Hispanics, and 1000 non-Hispanic White people with spontaneous $\mathrm{ICH}$ at 41 sites among 19 recruiting centers throughout the USA. In ERICH, ICH is defined as abrupt onset of severe headache, altered level of consciousness, or focal neurological deficit, associated with a focal blood collection within the brain parenchyma on brain imaging. This includes warfarin-associated $\mathrm{ICH}$ as well as peripartum ICH. Primary IVH was included in ERICH as well. ICH attributable to malignancies that led to coagulopathy, dural venous sinus thrombosis-associated hemorrhage, vascular malformations, aneurysms, tumors, or hemorrhagic conversion of recent ischemic stroke was excluded. To determine a patient's eligibility to be included in ERICH, study neurologists reviewed the patient's clinical presentation and neuroimaging. Clinical characteristics and demographics were prospectively collected through the abstraction of the hospital chart and through interviews with patients and surrogates by trained study investigators. Part of the standardized data collection protocol was the assessment of presenting signs and symptoms (i.e., symptoms that brought the patient in/seek treatment). Mental status at presentation was categorized as "alert and oriented", "drowsy/somnolent," "alert but confused," "coma/unresponsive," or "posturing." For the purpose of the present study, patients with a mental status at symptom onset assigned to the categories "coma/unresponsive" or "posturing" were defined as having loss of consciousness at ICH onset.

\section{Standard Protocol Approvals and Patient Consents}

Institutional review boards at each participating institution approved the ERICH study protocol. Written informed consent was obtained from each participant or legally authorized representative. A Spanish-language consent form was available for Spanish only speaking participants or for those who preferred the Spanish consent. Prior to an interview, the patient's capacity to give consent was screened using a consent comprehension questionnaire. If the patient failed this screen, a legally authorized representative was contacted for enrollment with priority to guardian/power of attorney, spouse, adult children, parents, and siblings, in that order.

\section{Neuroimaging}

Assessment of neuroimaging and determination of hematoma volumes in the ERICH cohort has been described previously [20-22]. Initial and follow-up CT images were used to obtain the following variables: ICH location and $\mathrm{ICH}$ and IVH volumes, using semiautomated computerized volumetric analysis (Alice software, Parexel Corporation, Waltham, MA). The presence of IVH was defined as an IVH volume measurement of $>0$. In patients with a follow-up CT scan, follow-up ICH volume was determined to assess hematoma expansion (HE). HE was 
defined as $\mathrm{a}>33 \%$ or $>6-\mathrm{mL}$ increase in hematoma volume between baseline and first follow-up scan [22]. ICH location was divided in the categories deep (basal ganglia or thalamus), lobar, brainstem, cerebellum, or primary IVH.

\section{Outcomes}

The primary endpoint was the presence of IVH on either admission or follow-up CT. For consistency between admission and follow up imaging, MRI was not considered in the present study. The secondary endpoint was unfavorable outcome 90 days after the index $\mathrm{ICH}$, defined as modified Rankin Scale (mRS) score of 4-6. Healthrelated quality of life (HRQoL) at 90 days was measured by the EQ-5D (on a scale of- $0.11-1$; higher values indicate better HRQoL) and EQ-5D visual analog scale (EQ$5 \mathrm{D}$ VAS; on a scale of $0-100$; higher values indicate better HRQoL) self-report questionnaires [23,24].

\section{Statistical Analysis}

Analyses were performed using SPSS version 27 (IBM, Armonk, NY, USA). Data are expressed as median and interquartile range (IQR), except for normally distributed data where we used mean and standard deviation (SD). For intergroup differences, we used Mann-Whitney U and $\chi^{2}$ tests, and binary logistic regression for associations between LOC and 1) IVH on admission CT or follow-up CT and 2) unfavorable outcome at 90 days. In the first binary logistic regression analysis, we included variables based on a combination of statistical significance on univariate analysis and clinical relevance. We included all variables that were significantly different between the two groups (IVH vs. no IVH, Table 2) with the exception of admission Glasgow coma scale (GCS) score (a potential association between admission GCS and IVH was tested in a separate analysis) and any information from prior medication use or past medical history, as these presumably are more difficult to assess accurately in patients with LOC (i.e., to avoid systematic bias). In the second logistic regression analysis, we included the original ICH score components as the most widely accepted variables determining outcome after ICH with the following important modifications: We substituted GCS for LOC and added HE as input variables [4,5,25]. The same analysis was repeated including GCS as covariate with different GCS dichotomization cutoffs.

\section{Mediation Analysis}

We performed a mediation analysis, using a singlemediator model, to determine whether IVH was a variable mediating any relationship between $\mathrm{LOC}$ at $\mathrm{ICH}$ onset (independent variable) and 3-month outcome (mRS, dependent variable). This method required the use of a continuous mediator variable. We therefore included IVH peak volume rather than IVH as a binary variable [26]. Results were quantified by reporting the mediation coefficients. For the effect of a given variable $\mathrm{X}$ on another variable $\mathrm{Y}$, the mediation coefficient demonstrates the change of $\mathrm{Y}$ if $\mathrm{X}$ changes by one unit. Of note, these coefficients are not proportional to each other, i.e., they cannot be compared directly between different regression analyses. To quantify the effect of LOC on clinical outcome without the mediated effect through $\mathrm{IVH}$, we calculated the variation quotient $\left(\mathrm{C}-\mathrm{C}^{\prime} / \mathrm{C}\right.$, with $\mathrm{C}$ being the total effect and $\mathrm{C}^{\prime}$ being the IVH-mediated effect of LOC on outcome).

For this analysis, we used the SPSS macro "process" version 3.5. In the mediation analysis, 95\% confidence intervals (CI) were bootstrapped, generated based on 5000 repetitions. The threshold of statistical significance was set at alpha $=0.05$.

\section{Results}

Among $3000 \mathrm{ICH}$ cases enrolled in the ERICH study, we excluded 144 cases because of missing CT volumetric measurements and 134 cases because they had a seizure at symptom onset. Baseline characteristics of included patients $(n=2724)$ are shown in Table 1. Those with LOC $(n=352)$ compared to those without LOC $(n=2372)$ were younger (mean 60 vs. 62 years, $p=0.005)$ and had a greater proportion of women among them ( 49 vs. $40 \%, p=0.04$ ). Patients with initial LOC had a lower median GCS score upon ED arrival (median 6 vs. $15, p<0.001$ ), greater admission systolic blood pressure (200 vs. $184 \mathrm{mmHg}, p<0.001)$, and serum glucose $(158$ vs. $127 \mathrm{mg} / \mathrm{dl}, p<0.001)$. Two hundred and seventy-one (77\%) patients with LOC had IVH on either admission or follow-up CT scan compared to 978 (41\%) patients without LOC $(p<0.001)$. Both initial ICH volume $(26$ vs. $10 \mathrm{ml}, p<0.001)$ and peak ICH volume (28 vs. $11 \mathrm{ml}$, $p<0.001)$ were significantly greater among patients with LOC compared to those without LOC. Characteristics of patients with the presence of IVH on either admission or follow-up CT scan compared to those without IVH on any CT scan are shown in Table 2. Table 3 summarizes hospital interventions, in-hospital mortality, 3-month functional (mRS), and quality of life outcomes (EQ5D) of patients stratified by the presence or absence of LOC at $\mathrm{ICH}$ onset. In-hospital mortality in patients with LOC was $38 \%$ compared to $7 \%$ in those without LOC $(p<0.001) .27 \%$ with LOC had unfavorable functional outcome at 3 months, compared to $23 \%$ in patients without initial LOC $(p<0.001)$. EQ5D at 3 months indicated significantly worse quality of life in patients with initial LOC in most categories, with the exceptions of pain, 
Table 1 Baseline characteristics of patients, stratified by initial loss of consciousness

\begin{tabular}{|c|c|c|c|}
\hline Characteristic $^{\mathrm{a}}$ & LOC present $(n=352)$ & LOC absent $(n=2372)$ & $p$ value \\
\hline Age, mean (SD), y & $60(15)$ & $62(14)$ & 0.005 \\
\hline Female & $171(49)$ & $958(40)$ & 0.04 \\
\hline Race & & & 0.3 \\
\hline White & $215(61)$ & $1539(65)$ & \\
\hline Black & $137(39)$ & $829(35)$ & \\
\hline Modified Rankin before ICH & $0(0-1)$ & $0(0-1)$ & 0.5 \\
\hline \multicolumn{4}{|l|}{ Medical history } \\
\hline Diabetes & $103(29)$ & $663(28)$ & 0.3 \\
\hline Hypertension & $281(80)$ & $1917(81)$ & 0.8 \\
\hline Elevated cholesterol & $90(26)$ & $760(32)$ & 0.07 \\
\hline$C A D$ & $34(10)$ & $318(13)$ & 0.1 \\
\hline AF & $25(7)$ & $230(10)$ & 0.2 \\
\hline TIA & $10(3)$ & $115(5)$ & 0.1 \\
\hline $\mathrm{CHF}$ & $22(6)$ & $175(7)$ & 0.6 \\
\hline \multicolumn{4}{|l|}{ Home medications } \\
\hline Aspirin & $57(16)$ & $651(27)$ & $<0.001$ \\
\hline Non-aspirin antiplatelet & $20(6)$ & $135(6)$ & 1.0 \\
\hline Warfarin & $26(7)$ & $216(9)$ & 0.3 \\
\hline Statin & $74(21)$ & $592(25)$ & 0.1 \\
\hline \multicolumn{4}{|l|}{ Clinical } \\
\hline Glucose value by EMS & $146(116-198)$ & $125(106-154)$ & $<0.001$ \\
\hline ED Glasgow Coma Score & $6(3-9)$ & $15(13-15)$ & $<0.001$ \\
\hline ED SBP & $200(167-226)$ & $184(158-213)$ & $<0.001$ \\
\hline \multicolumn{4}{|c|}{ Laboratory testing in emergency department } \\
\hline Serum glucose & $158(130-201)$ & $127(106-163)$ & $<0.001$ \\
\hline Hemoglobin & $13.6(12.2-15.1)$ & $13.8(12.6-15.0)$ & 0.2 \\
\hline Platelet count & $231(182-272)$ & $219(177-266)$ & 0.06 \\
\hline PTT & $27(25-30)$ & $29(26-32)$ & $<0.001$ \\
\hline First INR & $1.0(0.98-1.10)$ & $1.0(1.0-1.1)$ & 0.7 \\
\hline \multicolumn{4}{|l|}{ CT characteristics } \\
\hline Lobar hemorrhage location & $87(25)$ & $731(31)$ & 0.02 \\
\hline Initial CT ICH volume & $26(10-58)$ & $10(4-24)$ & $<0.001$ \\
\hline Peak ICH volume & $28(12-64)$ & $11(4-27)$ & $<0.001$ \\
\hline ICH expansion (> 33\%) & $36(16)$ & $275(17)$ & 0.7 \\
\hline IVH on any CT scan & $271(77)$ & $978(41)$ & $<0.001$ \\
\hline Peak IVH volume & $8(0.3-32)$ & $0(0-4)$ & $<0.001$ \\
\hline
\end{tabular}

$A F$ atrial fibrillation, $C A D$ coronary artery disease, $C H F$ congestive heart failure, $C T$ computed tomography, $E D$ emergency department, $E M S$ emergency medical services, ICH intracerebral hemorrhage, INR international normalized ratio, IVH intraventricular hemorrhage, LOC loss of consciousness, PTT partial thromboplastin time, SBP systolic blood pressure, SD Standard deviation, TIA transient ischemic attack, y year

a Data are represented as number (\%) or median (interquartile range), unless otherwise specified

anxiety, and depression, which were similar between both groups ( $p=0.09$ in both categories).

\section{LOC and IVH}

In order to determine whether LOC at ICH onset was independently associated with the presence of IVH on any in hospital CT scan, we conducted a multivariate regression analysis controlling for clinical variables differing between patients with and without IVH (Table 2).
LOC at ICH onset was independently associated with IVH occurrence (OR 2.6, CI 1.9-3.5) (Table 4). Exclusion of patients whose index ICH was located in the brainstem did not significantly alter this association (OR 2.8, CI 2.0-3.9), neither did exclusion of patients with positive drug screen on admission (OR 2.6, CI 1.6-3.9). We repeated this regression analysis substituting LOC at symptom onset with GCS $<8$ upon ED arrival, as 
Table 2 Baseline characteristics of patients, stratified by the presence versus absence of IVH on any hospital CT scan

\begin{tabular}{|c|c|c|c|}
\hline Characteristic $^{\mathrm{a}}$ & IVH present $(n=1249)$ & $\begin{array}{l}\text { IVH absent } \\
(n=1475)\end{array}$ & $p$ value \\
\hline Age, mean (SD), y & $62(14)$ & $62(14)$ & 1.0 \\
\hline Female & $520(42)$ & $609(41)$ & 0.9 \\
\hline Race & & & 0.048 \\
\hline White & $785(63)$ & $969(66)$ & \\
\hline Black & $464(37)$ & $502(34)$ & \\
\hline Modified Rankin score before ICH & $0(0-1)$ & $0(0-1)$ & 0.3 \\
\hline \multicolumn{4}{|l|}{ Medical history } \\
\hline Diabetes & $347(28)$ & $419(28)$ & 1.0 \\
\hline Hypertension & $1019(82)$ & $1179(80)$ & 0.06 \\
\hline Elevated cholesterol & $374(30)$ & $476(32)$ & 0.5 \\
\hline CAD & $162(13)$ & $190(13)$ & 0.8 \\
\hline$A F$ & $119(10)$ & $136(9)$ & 0.6 \\
\hline TIA & $60(5)$ & $65(4)$ & 0.5 \\
\hline $\mathrm{CHF}$ & $93(7)$ & $104(7)$ & 0.6 \\
\hline \multicolumn{4}{|l|}{ Home medications } \\
\hline Aspirin & $320(26)$ & $388(26)$ & 0.7 \\
\hline Non-aspirin antiplatelet & $75(6)$ & $80(5)$ & 0.5 \\
\hline Statin & $317(25)$ & $349(24)$ & 0.3 \\
\hline Warfarin & $112(9)$ & $130(9)$ & 0.9 \\
\hline \multicolumn{4}{|l|}{ Clinical } \\
\hline LOC at ICH onset & $271(22)$ & $81(6)$ & $<0.001$ \\
\hline Glucose value by EMS & $130(107-165)$ & $125(106-156)$ & 0.2 \\
\hline ED Glasgow Coma Score & $13(8-15)$ & $15(14-15)$ & $<0.001$ \\
\hline ED SBP & $190(163-218)$ & $180(155-212)$ & $<0.001$ \\
\hline ED DBP & $104(87-122)$ & $99(83-118)$ & $<0.001$ \\
\hline \multicolumn{4}{|c|}{ Laboratory testing in emergency department } \\
\hline Serum glucose & $140(116-177)$ & $122(104-161)$ & $<0.001$ \\
\hline Hemoglobin & $13.8(12.5-15.0)$ & $13.8(12.6-15.0)$ & 0.9 \\
\hline Platelet count & $221(177-270)$ & $220(177-263)$ & 0.4 \\
\hline PTT & $28(25-31)$ & $29(26-32)$ & $<0.001$ \\
\hline First INR & $1.0(1.0-1.1)$ & $1.0(1.0-1.1)$ & 0.6 \\
\hline \multicolumn{4}{|l|}{ CT characteristics } \\
\hline Initial CT ICH volume & $15(6-39)$ & $8(3-20)$ & $<0.001$ \\
\hline Peak ICH volume & $18(8-43)$ & $9(3-23)$ & $<0.001$ \\
\hline ICH expansion (> 33\%) & $155(12)$ & $156(11)$ & 0.9 \\
\hline Lobar hemorrhage location & $303(24)$ & $515(35)$ & $<0.001$ \\
\hline 3-month modified Rankin score & $3(2-4)$ & $2(1-3)$ & $<0.001$ \\
\hline
\end{tabular}

$A F$ atrial fibrillation, $C A D$ coronary artery disease, $C H F$ congestive heart failure, $C T$ computed tomography, $D B P$ diastolic blood pressure, $E D$ emergency department, EMS emergency medical services, ICH intracerebral hemorrhage, INR international normalized ratio, IVH intraventricular hemorrhage, LOC loss of consciousness, PTT partial thromboplastin time, SBP systolic blood pressure, SD Standard deviation, TIA transient ischemic attack, $y$ year

a Data are presented as number (\%) unless otherwise specified

assessed by ED staff showing a similarly strong association between GCS and IVH (OR 2.6, CI 1.9-3.5).

\section{LOC and Unfavorable Outcome at 90 days}

LOC at ICH onset was independently associated with unfavorable outcome at 90 days (OR 3.05, CI 1.96-4.75), controlling for $\mathrm{HE}$ and the components of the original
ICH score, but substituting GCS on ED admission for LOC (Table 5). Again, exclusion of brainstem ICH cases did not significantly alter this association (OR 3.34, CI 2.16-5.14), neither did exclusion of patients with positive drug screen (OR 3.9, CI 2.4-6.3). When adding GCS to the regression analysis, depending on the GCS 
Table 3 Hospital interventions, withdrawal of care and outcomes between patients with and without LOC

\begin{tabular}{|c|c|c|c|}
\hline Hospital interventions & LOC present $(n=352)$ & LOC absent $(n=2372)$ & $p$ value \\
\hline Craniotomy for clot evacuation & $44(13)$ & $170(7)$ & 0.19 \\
\hline ICP monitoring & $154(44)$ & $343(15)$ & $<0.001$ \\
\hline Intraventricular drain placed & $143(41)$ & $351(15)$ & $<0.001$ \\
\hline Shunt placement & $34(10)$ & $97(4)$ & $<0.001$ \\
\hline Intraventricular tPA & $19(5)$ & $31(8)$ & 0.01 \\
\hline \multicolumn{4}{|l|}{ Clinical outcome } \\
\hline In-hospital mortality & $132(38)$ & $163(7)$ & $<0.001$ \\
\hline Comfort measures only (CMO) & $97(28)$ & $187(8)$ & $<0.001$ \\
\hline 3-month mRS score & $4(3-5)$ & $3(1-4)$ & $<0.001$ \\
\hline 3-month unfavorable mRS (4-6) & $95(27)$ & $537(23)$ & $<0.001$ \\
\hline 3-month Barthel score & $50(10-90)$ & $90(55-100)$ & $<0.001$ \\
\hline EQ5D mobility & $2(2-3)$ & $2(1-2)$ & $<0.001$ \\
\hline EQ5D self-care & $2(2-3)$ & $2(1-2)$ & $<0.001$ \\
\hline EQ5D usual activities & $2(2-3)$ & $2(1-3)$ & $<0.001$ \\
\hline EQ5D pain & $2(1-2)$ & $2(1-2)$ & 0.09 \\
\hline EQ5D anxiety/depression & $2(1-2)$ & $1(1-2)$ & 0.09 \\
\hline EQ5D health state score & $60(44-70)$ & $70(50-80)$ & $<0.001$ \\
\hline
\end{tabular}

ICP intracranial pressure, $L O C$ loss of consciousness, $m R S$ modified Rankin score, $t P A$ tissue plasminogen activator

Table 4 Binary logistic regression analysis of the presence of IVH on any CT scan

\begin{tabular}{|c|c|c|c|}
\hline & OR & $95 \% \mathrm{Cl}$ & $p$ value \\
\hline First systolic BP in ED [mmHg] & 1.001 & $0.99-1.002$ & 0.46 \\
\hline Serum glucose upon ED admission & 1.001 & $1.0-1.003$ & 0.067 \\
\hline $\begin{array}{l}\text { Partial thromboplastin time upon ED } \\
\text { admission }\end{array}$ & 0.99 & $0.98-1.00$ & 0.052 \\
\hline Lobar ICH location & 0.30 & $0.23-0.38$ & $<0.001$ \\
\hline Peak ICH volume & 1.03 & $1.02-1.034$ & $<0.001$ \\
\hline Loss of consciousness at ICH onset & 2.6 & $1.90-3.54$ & $<0.001$ \\
\hline
\end{tabular}

$B P$ blood pressure, $C l$ confidence interval, $C T$ computed tomography, $E D$ emergency department, $I V H$ intraventricular hemorrhage, $O R$ odds ratio

Table 5 Binary logistic regression analysis of unfavorable outcome at 3 months (mRS 4-6). ICH score elements, hematoma expansion, and initial loss of consciousness as covariates

\begin{tabular}{lllc} 
& OR & $95 \% \mathrm{Cl}$ & $\boldsymbol{p}$ value \\
\hline Age $>80$ years & 3.5 & $2.333-5.154$ & $<0.001$ \\
Initial ICH volume $>30 \mathrm{ml}$ & 2.9 & $2.11-4.072$ & $<0.001$ \\
\hline Hematoma expansion* & 2.4 & $1.728-3.324$ & $<0.001$ \\
Infratentorial hemorrhage location & 1.03 & $0.688-1.545$ & 0.88 \\
\hline Loss of consciousness at ICH onset & 3.05 & $1.959-4.745$ & $<0.001$ \\
\hline
\end{tabular}

$\mathrm{Cl}$ confidence interval, $/ \mathrm{CH}$ intracerebral hemorrhage, $\mathrm{OR}$ odds ratio

*HE defined as a $>33 \%$ or $>6-\mathrm{mL}$ increase in hematoma volume between admission and follow-up CT
Table 6 Binary logistic regression analysis of unfavorable outcome at 3 months (mRS 4-6) controlling for ICH score elements and hematoma expansion (Table 5 ) as covariates and adding various GCS dichotomizations

\begin{tabular}{lllr} 
& OR & 95\%Cl & $p$ value \\
\hline GCS less than 15 & 2.8 & $2.166-3.650$ & $<0.001$ \\
\hline Loss of consciousness at ICH onset & 2.1 & $1.302-3.244$ & 0.002 \\
\hline GCS less than 13 & 3.7 & $2.685-5.097$ & $<0.001$ \\
\hline Loss of consciousness at ICH onset & 1.5 & $0.880-2.387$ & 0.145 \\
GCS less than 8 & 2.1 & $1.252-3.430$ & 0.005 \\
Loss of consciousness at ICH onset & 2.2 & $1.331-3.600$ & 0.002 \\
GCS less than 6 & 1.5 & $0.765-2.822$ & 0.248 \\
Loss of consciousness at ICH onset & 2.7 & $1.692-4.404$ & $<0.001$ \\
GCS 3 & 1.9 & $0.861-4.314$ & 0.11 \\
Loss of consciousness at ICH onset & 2.7 & $1.695-4.297$ & $<0.001$ \\
\hline
\end{tabular}

$\mathrm{Cl}$ confidence interval, GCS Glasgow coma scale, ICH intracerebral hemorrhage, $O R$ odds ratio

dichotomization cutoff, LOC was or was not associated with 90-day unfavorable outcome (Table 6).

The combination of discontinuation of aggressive medical care and do-not-resuscitate orders was placed in 9.9\% of study participants. Excluding these patients from the regression analysis, as shown in Table 5 , did not substantially change the association between LOC and 90-day outcome (OR 3.05, CI 1.96-4.76, analysis not shown).

\section{Mediation Analysis}

To test whether IVH was mediating a portion of the effect of $\mathrm{LOC}$ at ICH onset on outcome at 90 days, we regressed 
LOC and IVH onto unfavorable mRS at 90 days (dichotomized) together. The regression model revealed positive and statistically significant direct effects from LOC to IVH (beta $=11.62$, standard error $=1.04, p<0.001$ ), IVH to unfavorable outcome (beta $=0.02$, standard error $=0.003, p<0.001$ ), and LOC to unfavorable outcome (beta $=0.72$, standard error $=0.12, p<0.001$ ). The indirect effect of LOC to unfavorable outcome, mediated by IVH, was statistically significant (beta $=0.24$, bootstrapped standard error 0.04, bootstrapped CI $0.17-0.32)$. The variation quotient $\left(C-C^{\prime} / C\right)$ was $67 \%$. The results were similar in a sensitivity analysis in which we excluded patients whose index ICH was located in the brainstem. In this analysis, the indirect effect of LOC to unfavorable outcome, mediated by IVH, was similar to the one in the primary analysis (beta $=0.27$, bootstrapped standard error 0.04, bootstrapped CI 0.19-0.36, variation quotient $58 \%$ ).

\section{Discussion}

Among ICH cases from the multicenter case-control study ERICH, we found that loss of consciousness at the onset of $\mathrm{ICH}$ was independently associated with the presence of IVH on either admission or follow-up CT. LOC was also associated with 90-day unfavorable outcome when adjusting for components of the ICH score and HE. These findings held up in sensitivity analyses excluding patients with positive drug screening on hospital admission and those whose ICH was located in the brainstem. When controlling for GCS upon ED admission, the association between LOC and outcome was partially preserved depending on which GCS cutoff was used, suggesting overlapping prognostic information between LOC and admission GCS. A mediation analysis revealed that the association between LOC and outcome was significantly mediated through IVH. Because brainstem bleeds are in close proximity to the reticular activating system and the ventricular system, we conducted a sensitivity mediation analysis in which we excluded patients with brainstem bleeds. This analysis produced similar results as the primary analysis suggesting that the mediated effect of LOC on outcome through IVH was not driven or significantly attenuated by hematoma location in the brainstem. Overall, these findings suggest that in patients with ICH, LOC at symptom onset is a marker of IVH which in turn worsens outcome.

Prior studies have established that intraventricular extension of $\mathrm{ICH}$ is a time-dependent process which can occur with delay after a primarily parenchymal bleed and that in $7 \%-10 \%$ of all ICH cases IVH extension happens after the hospital admission CT scan $[4,5,9]$. With regard to its effect on outcome, IVH is not an "all or nothing" event. Increasing degrees of IVH correlate with an increasing risk of worse outcome [27-30]. These findings suggest that there might be a subgroup of patients with ICH in whom ICH is diagnosed early enough to potentially prevent IVH or contain an early stage IVH through blood pressure control or surgical intervention $[12,31]$. Certainly, such interventions would still need to be tested in prospective trials with regard to their efficacy. Despite the established relationship between IVH and clinical outcome, dedicated studies focusing on prediction of IVH were not available so far. Associations between greater ICH volume and hyperglycemia upon hospital arrival on the one hand and occurrence of IVH on the other have been reported. An inverse relationship between lobar hemorrhage location and IVH has been shown as well $[4,5,9,32]$. We reproduced the inverse relationship between lobar location of the $\mathrm{ICH}$ and IVH in our study. However, systolic BP and admission serum glucose were not associated with the presence of IVH in multivariate analysis (Table 4). Of note, several of the baseline characteristics of patients with LOC differed from those without LOC. Patients without LOC were older, more frequently male, more often had hyperlipidemia, more frequently were taking aspirin as home medication, and had greater PTT values on admission. Patients without IVH also had a greater median admission PTT. These differences might be driven by a greater prevalence of lobar ICH location in those without LOC (compared to those with LOC) and in those without IVH (compared to those with IVH). Patients with lobar ICH may have included a greater proportion of patients with amyloid angiopathy, a demographic that is typically older and has more comorbidities.

The novel finding our study adds is the identification of LOC as an acute clinical marker of IVH. LOC had the strongest independent association with IVH, and-being an early data point, collected before hospital arrivalLOC might be clinically more meaningful for early IVH prediction than the GCS score in the emergency department, or CT and laboratory findings which typically do not become available until hospital arrival. Our headto-head comparison of the pre-hospital LOC variable and the dichotomized GCS in the ED shows that LOC overpowers the GCS at lower cutoff values (less than 8) that tend to align more with "loss of consciousness." This suggests that the early level of consciousness after ICH is more meaningful for outcome prognostication than the assessment of consciousness in the ED, possibly because the pre-hospital level of consciousness is less likely confounded by sedating medications.

In our regression analysis (Table 4), we controlled for $\mathrm{ICH}$ volume and thus established that IVH is not simply a surrogate marker of a large parenchymal hematoma more likely to cause IVH and coma at symptom 
onset through mass effect [5]. However, we were not able to control for midline shift, hydrocephalus, or sudden increase in intracranial pressure (data not available), conditions that may co-occur with IVH and then lead to compression of brain structures associated with maintaining consciousness. We controlled for ICH location, but a greater anatomical resolution than large cohort studies typically provide would be needed to assess for hematoma locations as a primary cause for LOC. Studies investigating coma in patients with $\mathrm{ICH}$ have, among others, implicated brain structures with the presence of coma that are close to the ventricular system, for example lesions of the head of the caudate or the paramedian tegmentum [33,34]. In accordance with these findings from prior studies, patients with LOC had a greater proportion of deep and a smaller proportion of lobar hemorrhages in our study $(p=0.02)$. Thus, proximity of the hematoma to the ventricles may have influenced the association between impaired consciousness and IVH extension. Lastly, one may speculate that IVH may be an initial event with a cascade of downstream effects leading to LOC rather than being the direct cause of LOC. For example, IVH may cause hydrocephalus, which in turn may lead to ICP increase, which then might interfere with either cortical function or lead to different degrees and types of herniation. All of these mechanisms are well-described causes of coma $[35,36]$.

Although our article reports associations between clinical variables, which are relevant for diagnosis rather than therapy of IVH, it implicitly raises the question what the optimal intervention is in response to IVH detection. The literature reports conflicting findings about whether removal of blood and blood breakdown products or reversal of hydrocephalus should be the primary goal of surgical intervention [37-39]. The question is difficult to address in research studies as insertion of an external ventricular drain will typically treat both aspects of the pathology. The results of the CLEAR III trial suggest that intraventricular clot removal through insertion of an external ventricular drain in combination with alteplase irrigation does not generally improve clinical outcome in all patients with $\mathrm{ICH}$. However, it may be beneficial in selected subgroups or in combination with a lumbar drain [40-42]. More research is needed to define patient subgroups and interventions that target IVH.

The results of our study should be seen as preliminary and need confirmation in future prospective trials. If the association between LOC at ICH onset and IVH can be reproduced, its use in conjunction with other pre-hospital tests might allow efficient triage. Possibly, this might lead to earlier EVD insertion in those who need it.

Our study has limitations. First, although the study data were collected prospectively, our analysis was retrospective, which has conceptual limitations. We cannot exclude a degree of inaccuracy regarding assessment and documentation of the initial symptom (loss of consciousness); however, data in ERICH were collected carefully by dedicated study investigators. Second, the etiology and dynamics of the initial LOC were not addressed in this study. Importantly, data on the presence of hydrocephalus on CT were not available. Hyperacute onset hydrocephalus may contribute to the association between LOC and IVH, and it certainly contributes to the association between IVH and clinical outcome $[37,39]$. Many different trajectories after initial LOC are possible, ranging from recovery to progression to brain death. Future studies will hopefully clarify these different trajectories as well as how initial LOC relates to early and delayed neurological deterioration. Third, we did not differentiate between patients with initial and delayed IVH in our analysis. The distinction between the two depends on the time interval between symptom onset and imaging [5], a variable that was not available with hour/minute resolution for all cases in ERICH. To account for that, we decided to analyze patients with initial and delayed IVH together, and to use, wherever available, peak ICH volume instead of $\mathrm{ICH}$ volume on admission CT. Peak $\mathrm{ICH}$ volume can be assumed to be a more robust measure in the context of variable symptom-to-imaging time intervals. Of note, around 30\% of ERICH cases did not have follow-up CT imaging. Thus, in these cases only one $\mathrm{ICH}$ volume measurement is available which precludes assessment of HE. Dedicated studies would be needed to assess whether LOC or decline of mental status after hospital arrival is associated with delayed IVH.

Fourth, there are limitations inherent to mediation analysis as a statistical technique; mediation analysis may help to identify statistical associations, but it cannot prove causality between mediator and dependent variable. Fifth, this is a clinical study and correlations between clinical and imaging findings cannot be made with high accuracy. Small prospective studies with highresolution imaging technology might be more appropriate study designs for this purpose. Lastly, the ERICH study includes only people of White or Black race (both non-Hispanic) or Hispanic ethnicity which may limit the generalizability to other populations.

\section{Conclusions}

In this analysis of $\mathrm{ICH}$ cases from the prospective multicenter ERICH cohort, pre-hospital LOC was strongly associated with the presence of IVH on admission or follow-up CT brain scan. This information may become clinically useful for patient triage and risk stratification in future trials. LOC was also associated with long-term functional outcome after $\mathrm{ICH}$, but a significant portion 
of this effect was mediated through IVH. The findings of this report emphasize the importance of IVH as a treatment target in patients with ICH. Future studies are needed to characterize how LOC can be integrated into clinical tools that predict IVH, and how IVH and LOC may connect mechanistically.

\begin{abstract}
Author details
1 Department of Neurology, Yale School of Medicine, 15 York Street, LLCl 10th floor, Room 1003C, P.O. Box 208018, New Haven, CT 06520, USA. ${ }^{2}$ Department of Neurology, Clinical and Translational Neuroscience Unit, Feil Family Brain and Mind Research Institute, Weill Cornell Medical College, New York, NY, USA. ${ }^{3}$ Department of Neurosurgery, Yale School of Medicine, New Haven, CT, USA. ${ }^{4}$ Klinik Und Hochschulambulanz Für Neurologie, Charité-Universitätsmedizin, Berlin, Germany. ${ }^{5}$ Department of Neurology and Rehabilitation Medicine, University of Cincinnati, Cincinnati, OH, USA.
\end{abstract}

\section{Acknowledgements}

We thank Dr. Daniel Golkowski (Technische Universität München, Germany) for helpful discussions on mediation analysis. We also thank the patients and their relatives for their willingness to participate in the research study.

\begin{abstract}
Author Contributions
The authorship requirements have been met and the final manuscript was approved by all authors. JW developed the project, carried out the statistical analysis, and wrote the manuscript. GJF interpreted the results and edited the manuscript. ACL interpreted the results and edited the manuscript. CM interpreted the results and edited the manuscript. ME interpreted the results and edited the manuscript. LS interpreted the results and edited the manuscript. DW was involved in the data collection process, contributed to the study concept, interpreted the results and edited the manuscript. KNS provided study supervision, contributed to the study concept, interpreted the results and edited the manuscript.
\end{abstract}

\section{Source of Support}

There was no targeted funding for this study. Dr. Sheth received funding from the NIH (U24NS107215, U24NS107136, U01NS106513, RO1NR018335, R03NS1 12859, R01NS110721) and AHA (17CSA33550004). Ms. Leasure was supported by the AHA Medical Student Research Fellowship.

\section{Conflict of interest}

Dr. Witsch reports to be an editorial team member of the Resident \& Fellow section of Neurology. Dr. Falcone has nothing to disclose. Ms. Leasure has nothing to disclose. Dr. Matouk has nothing to disclose. Dr. Endres reports grants from Bayer, grants from German Research Foundation (DFG), grants from German Federal Ministry of Education and Research (BMBF), grants from German Center for Neurodegenerative Diseases (DZNE), grants from German Center for Cardiovascular Research (DZHK), grants from European Union, grants from Corona Foundation, and grants from Fondation Leducq, outside the submitted work. Dr. Sansing reports grants from NIH, non-financial support from Genentech, outside the submitted work. Dr. Woo reports grants from $\mathrm{NIH}$, during the conduct of the study. Dr. Sheth reports grants from $\mathrm{NIH}$, grants from AHA, grants from Novartis, grants from Biogen, grants from Bard, grants from Hyperfine, and grants from Zoll, outside the submitted work.

\section{Ethical Approval}

All procedures performed in the studies involving humans were in accordance with the ethical standards of the national research committee and with the 1964 Helsinki declaration and its later amendments. Institutional review boards at each participating institution approved the Ethnic/Racial Variations of Intracerebral Hemorrhage study (ERICH, NS069763) study protocol.

\section{Open Access}

This article is licensed under a Creative Commons Attribution 4.0 International License, which permits use, sharing, adaptation, distribution and reproduction in any medium or format, as long as you give appropriate credit to the original author(s) and the source, provide a link to the Creative Commons licence, and indicate if changes were made. The images or other third party material in this article are included in the article's Creative Commons licence, unless indicated otherwise in a credit line to the material. If material is not included in the article's Creative Commons licence and your intended use is not permitted by statutory regulation or exceeds the permitted use, you will need to obtain permission directly from the copyright holder. To view a copy of this licence, visit http://creativecommons.org/licenses/by/4.0/.

\section{Publisher's Note}

Springer Nature remains neutral with regard to jurisdictional claims in published maps and institutional affiliations.

Received: 14 July 2020 Accepted: 15 December 2020

Published online: 21 January 2021

\section{References}

1. Marini S, Crawford K, Morotti A, et al. Association of apolipoprotein E with intracerebral hemorrhage risk by race/ethnicity: a meta-analysis. JAMA Neurol. 2019;76:480-91.

2. Lioutas VA, Beiser AS, Aparicio HJ, et al. Assessment of incidence and risk factors of intracerebral hemorrhage among participants in the framingham heart study between 1948 and 2016. JAMA Neurol. 2020;77:1252.

3. Daverat P, Castel JP, Dartigues JF, Orgogozo JM. Death and functional outcome after spontaneous intracerebral haemorrhage. a prospective study of 166 cases using multivariate analysis. Stroke. 1991;22(1):1-6.

4. Maas MB, Nemeth AJ, Rosenberg NF, et al. Delayed intraventricular hemorrhage is common and worsens outcomes in intracerebral hemorrhage. Neurology. 2013;80(14):1295-9.

5. Witsch J, Bruce E, Meyers E, et al. Intraventricular hemorrhage expansion in patients with spontaneous intracerebral hemorrhage. Neurology. 2015;84(10):989-94

6. Phan TG, Koh M, Vierkant RA, Wijdicks EF. Hydrocephalus is a determinant of early mortality in putaminal hemorrhage. Stroke. 2000;31(9):2157-62.

7. Cordonnier C, Demchuk A, Ziai W, Anderson CS. Intracerebral haemorrhage: current approaches to acute management. Lancet. 2018;392(10154):1257-68

8. You S, Zheng D, Delcourt C, et al. Determinants of early versus delayed neurological deterioration in intracerebral hemorrhage. Stroke. 2019;50(6):1409-14.

9. Moullaali TJ, Sato S, Wang X, et al. Prognostic significance of delayed intraventricular haemorrhage in the INTERACT studies. J Neurol Neurosurg Psychiatry. 2017:88(1):19-24.

10. Li Q, Li R, Zhao $L B$, et al. Intraventricular hemorrhage growth: definition prevalence and association with hematoma expansion and prognosis. Neurocrit Care. 2020;33:732-9.

11. Soun JE, Montes $\mathrm{D}$, Yu F, et al. Spot sign in secondary intraventricular hemorrhage predicts early neurological decline. Clin Neuroradiol. 2019:30(4):761-8.

12. Leasure AC, Qureshi Al, Murthy SB, et al. Association of intensive blood pressure reduction with risk of hematoma expansion in patients with deep intracerebral hemorrhage. JAMA Neurol. 2019;76(8):949.

13. Mayer SA, Brun NC, Begtrup K, et al. Recombinant activated factor VII for acute intracerebral hemorrhage. N Engl J Med. 2005;352(8):777-85.

14. Helwig SA, Ragoschke-Schumm A, Schwindling L, et al. prehospital stroke management optimized by use of clinical scoring vs mobile stroke unit for triage of patients with stroke a randomized clinical trial. JAMA Neurol. 2019;76(12):1484

15. Luger S, Witsch J, Dietz A, et al. Glial fibrillary acidic protein serum levels distinguish between intracerebral hemorrhage and cerebral ischemia in the early phase of stroke. Clin Chem. 2017:63(1):377-85.

16. Rozanski M, Waldschmidt C, Kunz A, et al. Glial fibrillary acidic protein for prehospital diagnosis of intracerebral hemorrhage. Cerebrovasc Dis. 2017:43(1-2):76-81

17. Perry LA, Lucarelli T, Penny-Dimri JC, et al. Glial fibrillary acidic protein for the early diagnosis of intracerebral hemorrhage: Systematic review and meta-analysis of diagnostic test accuracy. Int J Stroke. 2019;14(4):390-9. 
18. Suwatcharangkoon S, Meyers E, Falo C, et al. Loss of consciousness at onset of subarachnoid hemorrhage as an important marker of early brain injury. JAMA Neurol. 2016;73(1):28-35.

19. Woo D, Rosand J, Kidwell C, et al. The ethnic/racial variations of intracerebral hemorrhage (ERICH) study protocol. Stroke. 2013;44(10):e120-5.

20. James ML, Langefeld CD, Sekar P, et al. Assessment of the interaction of age and sex on 90-day outcome after intracerebral hemorrhage. Neurology. 2017;89(10):1011-9.

21. Marini S, Devan WJ, Radmanesh F, et al. 17p12 Influences hematoma volume and outcome in spontaneous intracerebral hemorrhage. Stroke. 2018;49(7):1618-25.

22. Uniken Venema SM, Marini S, Brouwers HB, et al. Associations of radiographic cerebral small vessel disease with acute intracerebral hemorrhage hematoma expansion and intraventricular hemorrhage. Neurocrit Care. 2019;32(2):383-91.

24. EuroQol--A new facility for the measurement of health-related quality of life. Health Policy 199016(3): 199-208.

24. Chen CJ, Ding D, Ironside N, et al. Intracranial pressure monitoring in patients with spontaneous intracerebral hemorrhage. J Neurosurg. 2019;50:1-11.

25. Hemphill JC 3rd, Bonovich DC, Besmertis L, Manley GT, Johnston SC. The ICH score: a simple, reliable grading scale for intracerebral hemorrhage. Stroke. 2001;32(4):891-7.

26. MacKinnon DP, Fairchild AJ, Fritz MS. Mediation analysis. Annu Rev Psychol. 2007:58:593-614.

27. Tuhrim S, Horowitz DR, Sacher M, Godbold JH. Validation and comparison of models predicting survival following intracerebral hemorrhage. Crit Care Med. 1995;23(5):950-4

28. Tuhrim S, Horowitz DR, Sacher M, Godbold JH. Volume of ventricular blood is an important determinant of outcome in supratentorial intracerebral hemorrhage. Crit Care Med. 1999;27(3):617-21.

29. Morgan TC, Dawson J, Spengler D, et al. The Modified Graeb Score: an enhanced tool for intraventricular hemorrhage measurement and prediction of functional outcome. Stroke. 2013;44(3):635-41.

30. Yogendrakumar $V$, Ramsay T, Fergusson D, et al. New and expanding ventricular hemorrhage predicts poor outcome in acute intracerebral hemorrhage. Neurology. 2019;93(9):e879-88.

31. Hanley DF, Thompson RE, Rosenblum M, et al. Efficacy and safety of minimally invasive surgery with thrombolysis in intracerebral haemorrhage evacuation (MISTIE III) a randomised, controlled, open-label, blinded endpoint phase 3 trial. Lancet. 2019;393:1021-32.

32. Appelboom G, Piazza MA, Hwang BY, et al. Severity of intraventricular extension correlates with level of admission glucose after intracerebral hemorrhage. Stroke. 2011;42(7):1883-8.

33. Rohaut B, Doyle KW, Reynolds AS, et al. Deep structural brain lesions associated with consciousness impairment early after hemorrhagic stroke. Sci Rep. 2019;9(1):4174

34. Giacino JT, Fins JJ, Laureys S, Schiff ND. Disorders of consciousness after acquired brain injury: the state of the science. Nat Rev Neurol. 2014;10(2):99-114

35. Staykov D, Volbers B, Wagner I, et al. Prognostic significance of third ventricle blood volume in intracerebral haemorrhage with severe ventricular involvement. J Neurol Neurosurg Psychiatry. 2011;82(11):1260-3.

36. Posner JB, Saper CB, Schiff ND, Claassen J. Plum and posner's diagnosis and treatment of stupor and coma. England: Oxford University Press; 2019.

37. Diringer MN, Edwards DF, Zazulia AR. Hydrocephalus: a previously unrecognized predictor of poor outcome from supratentorial intracerebral hemorrhage. Stroke. 1998;29(7):1352-7.

38. Bhattathiri PS, Gregson B, Prasad KS, Mendelow AD, Investigators S. Intraventricular hemorrhage and hydrocephalus after spontaneous intracerebral hemorrhage: results from the STICH trial. Acta Neurochir Suppl. 2006;96:65-8.

39. Hughes JD, Puffer R, Rabinstein AA. Risk factors for hydrocephalus requiring external ventricular drainage in patients with intraventricular hemorrhage. J Neurosurg. 2015;123(6):1439-46.

40. Hanley DF, Lane K, McBee N, et al. Thrombolytic removal of intraventricular haemorrhage in treatment of severe stroke: results of the randomised, multicentre, multiregion, placebo-controlled CLEAR III trial. Lancet. 2017:389(10069):603-11.

41. Ullman NL, Tahsili-Fahadan P, Thompson CB, Ziai WC, Hanley DF. Third ventricle obstruction by thalamic intracerebral hemorrhage predicts poor functional outcome among patients treated with alteplase in the CLEAR III Trial. Neurocrit Care. 2019;30(2):380-6.

42. Staykov D, Kuramatsu JB, Bardutzky J, et al. Efficacy and safety of combined intraventricular fibrinolysis with lumbar drainage for prevention of permanent shunt dependency after intracerebral hemorrhage with severe ventricular involvement: a randomized trial and individual patient data meta-analysis. Ann Neurol. 2017;81(1):93-103. 